\title{
Circadian Preference, Sleep Quality, and Health-impairing Lifestyles Among Undergraduates of Medical University
}

Anil Gangwar ${ }^{1}$, Sunita Tiwari ${ }^{2}$, Anita Rawat ${ }^{3}$, Ajay Verma ${ }^{4}$, Kalpana Singh ${ }^{5}$, Surya Kant ${ }^{6}$, Ravindra Kumar Garg ${ }^{7}$, Prithvi K. Singh ${ }^{8}$

1. Physiology, King George Medical University, Lucknow, IND 2. Department of Physiology, King George Medical University Lucknow,INDIA 3. Department of Physiology, Hind Institute of Medical Sciences,sitapur, India 4. Respiratory Medicine, King George's Medical University, Lucknow, IND 5. Department of Biochemistry, King George Medical University Lucknow,INDIA, Lucknow, IND 6. Department of Respiratory Medicine, King George Medical University Lucknow,INDIA 7. Department of Neurology, King George Medical University, Lucknow, IND 8. Center for Advance Research/Cytogenetic Unit, King George Medical University, Lucknow, IND

$\square$ Corresponding author: Sunita Tiwari, sunita_kgmu@yahoo.com

Disclosures can be found in Additional Information at the end of the article

\section{Abstract}

Background/Aims

Health-impairing lifestyle during adolescence is an important marker for poor health. An unhealthy lifestyle may lead to physical and psychological problems in adulthood. Most of the prior researches were done among the adult population. Therefore, we assessed the chronotype pattern and correlation of health-impairing lifestyles with sleep quality according to circadian typology in medical students.

\section{Material and Methods}

In this cross-sectional research, a total of 203 subjects were enrolled. All subjects were divided into definite evening chronotype $(n=73)$, intermediate chronotype $(n=87)$, and definite morning chronotype $(n=43)$. Electronic media use at bedtime and duration of media use, the timing of dinner, smoking, tobacco chewing, and alcohol consumption were assessed with the help of a preformed proforma. Physical activity, sleep quality, daytime sleepiness, and chronotype were assessed by International Physical Activity Questionnaire (IPAQ), Pittsburgh Sleep Quality Index (PSQI), Epworth Sleepiness Score (ESS), and Morningness-Eveningness Questionnaire Self-assessment version (MEQ-SA), respectively.

\section{Results}

Subjects of the evening chronotype were suffering more with poor sleep quality. Evening chronotype had a significant $(\mathrm{p}<0.05)$ positive correlation between poor sleep quality and sex, tobacco smoking, alcohol drinking, type of diet, and timing of dinner.

Conclusion

Circadian typology demonstrated the significant correlation of health-impairing lifestyles with sleep quality. From this observation, it might be a better way to plan their daily activities, in accordance with their chronotypes, benefiting not only their academic performance but 
also their quality of life.

Categories: Medical Education, Public Health, Epidemiology/Public Health

Keywords: chronotype, medical students, epworth sleepiness score, morningness-eveningness, sleep quality

\section{Introduction}

The relationship between psychological and behavioral variables with different sleep-wake habits was studied in human beings [1]. Morningness-eveningness refers to diurnal preferences of an individual for activity and alertness in the morning and evening. Various biological, genetic and psychosocial components are associated with the morningness-eveningness [2]. Initially, the Morningness-Eveningness Questionnaire [3] was used to distinguish between the two extreme diurnal types: morning type and evening type [4], and since then this questionnaire has been modified a number of times [5]. Some research involved morning type and evening type [3], whereas in other research, morning, intermediate, and evening types were studied [6]. Some important factors such as age, sex [7-8], personality [9], food intake [10], sleeping habits, smoking [11] and physical activity [12] were associated with morningnesseveningness. Previous studies have found that in adults [13] and adolescents [14-15] both circadian typology were associated with tobacco smoking. It was found that individuals with eveningness chronotypes have higher tendency to consume psychoactive substances as compared morningness and intermediate chronotype [10]. Some studies have found that subjects with eveningness chronotype have a higher tendency of having health-impairing lifestyle compared to morningness chronotype [12, 15]. However, the majority of these studies involved adult subjects. Therefore, it would be important to focus on the adolescent population in order to understand the associations between chronotype and health impairing lifestyle in a younger population. Regardless of the individual chronotype, the sleep-wake cycle should be synchronized in every individual. In undergraduates of the medical university, sleep-wake cycle is modified by various factors, such as curricular load, extracurricular activities, physical and emotional stress and pressure for high academic performance [16]. Therefore, it is necessary to focus on this subset of adolescents. The aims of this study were (a) to identify chronotypes pattern in medical undergraduates empirically, (b) to test the correlation between sleep quality and health impairing lifestyle. Based on our literature review, our hypothesis was that undergraduates of a medical university with morningness chronotype would be associated with lower likelihood of smoking, alcohol use, and electronic media use.

This research work was presented at Int. Conf. on Sleep Medicine and Research (Goasleep2017), Dona Paula, India, Sept. 22-23, 2017 (Gangwar AK, Rawat A, Tiwari S, Garg R, Kant S: Correlation of Chronotype with Sleep Quality, Demographic and Life Style Factors in Asymptomatic Healthy Adolescents - poster 38; http://link.springer.com/content/pdf/10.1007/s41782-017-0013-x.pdf.)

\section{Materials And Methods}

In this cross-sectional study, a total of 203 subjects were enrolled among first-year medical undergraduates on the basis of inclusion and exclusion criteria. Subjects with any known sleep problem, oro-nasal disease, head injury, and not willing to participate in the study were excluded from the study. Subjects with any chronic illness, such as diabetes mellitus, hypertension, and chronic respiratory disease, were also excluded from the study. All subjects were divided into three groups: group 1 (definite evening chronotype, n=73), group 2 (intermediate chronotype, $n=87$ ), and group 3 (definite morning chronotype, $n=43$ ) on the basis of the Morningness-Eveningness score [3]. Informed written consent was taken from all subjects after ethical clearance by the ethical committee of King George's Medical University 


\section{Cureus}

(KGMU), Lucknow, India. The participants were then invited to respond to questionnaires. Complete clinical evaluation of subjects was done on the basis of a preformed proforma. Anthropometric measurements, such as height, weight, body mass index (BMI), and neck circumference, were taken by trained nursing staff. Type of electronic media used, whether used at bedtime or not, and duration of electronic media use (hours per week) were assessed on a subjective basis. Type of food (vegetarian or non-vegetarian), the timing of dinner, tobacco smoking, tobacco chewing, and alcohol consumption were also assessed subjectively with the help of the preformed proforma. The level of physical activity, sleep quality, daytime sleepiness, and chronotype were assessed by International Physical Activity Questionnaire (IPAQ) [17], Pittsburgh Sleep Quality Index (PSQI) [18], Epworth Sleepiness Score (ESS) [19], and Morningness-Eveningness Questionnaire Self-assessment version (MEQ-SA) [3], respectively. The Statistical Package for Social Sciences (SPSS) (IBM SPSS Statistics, Armonk, NY), version 21 was used for data analysis. Data were summarized as the mean \pm standard deviation (SD) and the crude odds ratios (OR) of the demographic and lifestyle factors of all study subjects were calculated for poor sleep quality. Karl Pearson's correlation coefficient between demographic and lifestyle factors with poor sleep quality was calculated for all the three groups.

\section{Results}

Demographic and factors affecting the lifestyles of groups 1, 2, and 3 are summarized in Table 1. No statistically significant difference was observed in age, sex, weight, height, neck circumference, tobacco chewing, tobacco smoking, alcohol drinking, physical activity, and duration of electronic media use (hours per week) among the study groups, although a statistically significant difference was found in BMI ( $<<0.029)$, sleep quality $(p<0.001)$, sleep duration $(p<0.001)$, electronic media use at bedtime $(p<0.004)$, and timing of dinner $(p<$ 0.001 ) among study groups. On comparing the groups, we saw that subjects of group 1 were suffering more with poor sleep quality compared to subjects of groups 2 and 3 .

\begin{tabular}{|c|c|c|c|c|}
\hline & Group 1 (n = 73) & Group 2 (n = 87) & Group 3 (n = 43) & p-Value \\
\hline Age (years) & $18.21 \pm 0.67$ & $18.90 \pm 0.68$ & $18.05 \pm 0.65$ & 0.395 \\
\hline \multicolumn{5}{|l|}{ Sex } \\
\hline Male & $47(64.38 \%)$ & 57 (65.52\%) & $27(62.79 \%)$ & \multirow{2}{*}{0.954} \\
\hline Female & $26(35.62 \%)$ & $30(34.48 \%)$ & $16(37.21 \%)$ & \\
\hline Weight (kg) & $64.49 \pm 8.53$ & $63.62 \pm 8.35$ & $61.95 \pm 8.30$ & 0.293 \\
\hline Height (cm) & $167.27 \pm 8.17$ & $168.02 \pm 8.17$ & $168.21 \pm 8.57$ & 0.793 \\
\hline BMI & $23.06 \pm 2.45$ & $22.50 \pm 2.15$ & $21.89 \pm 2.27$ & $0.029^{*}$ \\
\hline Neck Circumference (cm) & $37.41 \pm 2.40$ & $37.47 \pm 2.36$ & $37.30 \pm 2.58$ & 0.933 \\
\hline \multicolumn{5}{|l|}{ Sleep Quality } \\
\hline Good & $7(9.6 \%)$ & 19 (21.84\%) & 26 (60.46\%) & \multirow{2}{*}{$<0.001^{*}$} \\
\hline Poor & $66(90.4 \%)$ & $68(78.16 \%)$ & $17(39.54 \%)$ & \\
\hline \multicolumn{5}{|l|}{ Sleep Duration (hrs) } \\
\hline$\geq 7 \mathrm{hrs}$ & $8(10.99 \%)$ & $10(11.5 \%)$ & $10(23.25 \%)$ & \\
\hline
\end{tabular}




\section{Cureus}

$6-7$ hrs

$<6 \mathrm{hrs}$

Tobacco Smoking

Yes

No

Tobacco Chewing

Yes

No

Alcohol Drinking

Yes

No

Physical Activity

Inactive

Minimally active

HEPA active (Health enhancing physical activity)

Electronic Media Use (hrs/wk)

Electronic Media Used (At bedtime)

Yes

No

Timing of Dinner

$<9$ PM

$\geq 9$ PM
$15(20.55 \%)$

$50(68.5 \%)$

$16(18.4 \%)$

$61(70.1 \%)$

25 (58.14\%)

$<0.001^{*}$

$8(18.6 \%)$

$30(41.1 \%)$

$30(34.48 \%)$

$10(23.25 \%)$

$43(58.9 \%)$

$57(65.52 \%)$

$33(76.75 \%)$

0.148

$4(5.48 \%)$

$5(5.75 \%)$

$1(2.33 \%)$

$69(94.52 \%)$

$82(94.25 \%)$

$42(97.67 \%)$

0.672

$30(41.1 \%)$

33 (37.93\%)

$9(20.93 \%)$

$43(58.9 \%)$

$54(62.07 \%)$

$34(79.07 \%)$

$2(2.74 \%)$

$1(1.15 \%)$

$1(2.32 \%)$

$61(83.56 \%)$

$72(82.76 \%)$

$34(79.07 \%)$

0.904

$10(13.69 \%)$

$14(16.09 \%)$

$8(18.6 \%)$

$18.87 \pm 5.85$

$20.45 \pm 5.99$

$18.85 \pm 5.98$

0.174

$57(78.1 \%)$

$71(81.61 \%)$

$16(18.39 \%)$

$16(37.21 \%)$

27 (62.79\%)

$<0.001^{*}$

$16(21.9 \%)$

$18(24.66 \%)$

$51(58.62 \%)$

$28(65.12 \%)$

$55(75.34 \%)$

$36(41.38 \%)$

$15(34.88 \%)$

\section{TABLE 1: Demographic and Lifestyle Factors of Study Subjects}

Data are represented as mean \pm standard deviation, $\mathrm{n}(\%)$, and ratio.

* significant ( $p<0.05)$; cm: centimetre; hrs: hours; kg: kilograms; n: number; SD: standard deviation; wk: week

Crude OR of demographic and lifestyle factors for poor sleep quality are shown in Table 2. All the subjects with an age $>20$ years showed high OR compared to subjects with an age $\leqslant 20$ years for poor sleep quality. Short sleep duration (<6 hours) showed a high OR of 1.24 (0.75 - 2.04), while long sleep duration ( $\geqslant 7$ hours) showed a lower OR of $0.94(0.45-1.97)$ for poor sleep quality. The long duration of electronic media use (> 14 hours/ week) and electronic media use at bedtime showed higher OR compared to less duration of electronic media use ( $\leqslant 7$ hours/ 


\section{Cureus}

week) and electronic media not used at bedtime, respectively. Late night dinner ( $\geqslant 9 \mathrm{pm})$ was strongly associated with poor sleep quality compared to dinner taken before $9 \mathrm{pm}$. A high ESS was strongly associated with poor sleep quality compared to a low ESS.

\section{Poor Sleep Quality Frequency (\%)}

40.39

32.51

Neck Circumference $(\mathrm{cm})$

Male

$<37$

$\geq 37$

Female

$<34$

$\geq 34$

Physical Activity

Inactive

Minimally Active

HEPA

Sleep Duration (hrs)

$\geq 7$

$6-7$

$<6$

EM used (hrs/week)

$\leq 7$

$8-14$

$>14$

EM Used at Bedtime

Not Used

Used

Timing of Dinner

$<9$ PM
46.80

62.07

14.78

58.13

31.03
OR (Cl)

p-Value

$1.03(0.67-1.58) \quad 0.975$

$1.09(0.61-1.97) \quad 0.844$

$0.90(0.36-2.27) \quad 0.823$

$0.97(0.21-4.40) \quad 0.964$

$1.00(0.20-4.90) \quad 1$

$0.94(0.45-1.97) \quad 0.879$

$1.24(0.75-2.04) \quad 0.467$

1

$1.33(0.32-4.07) \quad 0.847$

$1.79(0.38-3.69) \quad 0.777$

1

$1.32(0.79-2.20) \quad 0.349$ 


\section{Cureus}

$\geq 9$ PM

41.87

$1.24(0.81-1.89)$

ESS

$0-9$

$1.53(0.78-3.04)$
$1.53(0.84-2.79)$

0.29

12.81
50.74

9.36

1

$12-24$

\section{TABLE 2: Crude Odds Ratios (OR) of Demographic and Lifestyle Factors for Poor Sleep Quality}

$\mathrm{Cl}$ : confidence interval; cm: centimetre; EM: electronic media; ESS: Epworth Sleepiness Score; HEPA: health-enhancing physical activity; hrs: hours; P: probability

Correlation between demographic and lifestyle factors with poor sleep quality in groups 1, 2, and 3 are shown in Table 3 . There was a significant positive correlation between poor sleep quality and sex, tobacco smoking, alcohol drinking, diet (vegetarian or non-vegetarian), and timing of dinner. There was a significant negative correlation between poor sleep quality and age and electronic media use duration (hours/ week). Weight, height, BMI, and neck circumference have a negative correlation with poor sleep quality but statistically were not significant in the subjects of group 1. There was a significant positive correlation between poor sleep quality and age, sex, tobacco smoking, alcohol drinking, diet, electronic media use duration, and ESS. Other demographic and lifestyle factors were positively correlated with poor sleep quality but statistically were not significant in the subjects of group 2 . There was a significant positive correlation between poor sleep quality and age, sex, and tobacco smoking but no association was present between poor sleep quality and tobacco chewing, alcohol drinking, and electronic media use at bedtime. Weight, height, the timing of dinner, neck circumference, sleep duration, and electronic media use duration were negatively correlated with poor sleep quality but the correlation was not statistically significant in the subjects of group 3 . 


\section{Cureus}

\begin{tabular}{|llll|}
$\begin{array}{l}\text { Demographic and } \\
\text { lifestyle variable }\end{array}$ & $\begin{array}{l}\text { Group 1 (Karl-Pearson's } \\
\text { correlation coefficient) }\end{array}$ & $\begin{array}{l}\text { Group 2 (Karl-Pearson's } \\
\text { correlation coefficient) }\end{array}$ & $\begin{array}{l}\text { Group 3 (Karl-Pearson's } \\
\text { correlation coefficient) }\end{array}$ \\
\hline Age & $-0.333^{*}$ & $0.445^{*}$ & $0.739^{*}$ \\
Sex & $0.885^{*}$ & $0.785^{*}$ & $0.511^{*}$ \\
Weight & -0.163 & 0.113 & -0.112 \\
Height & -0.155 & 0.028 & -0.159 \\
BMI & -0.040 & 0.099 & 0.024 \\
Smoking & $0.508^{*}$ & $0.446^{*}$ & $0.511^{*}$ \\
Tobacco & 0.214 & 0.136 & 0 \\
Alcohol & $0.427^{*}$ & $0.447^{*}$ & 0 \\
Diet & $0.535^{*}$ & $0.374^{*}$ & 0.366 \\
Timing of Dinner & $0.261^{*}$ & 0.033 & -0.198 \\
Neck Circumference & -0.244 & 0.067 & -0.391 \\
Physical Activity & 0.071 & 0.079 & 0.276 \\
Sleep Duration & 0.149 & 0.178 & -0.130 \\
EM used (hrs/wk) & $-0.361^{*}$ & $0.377^{*}$ & -0.061 \\
EM at bedtime & 0.205 & 0.111 & 0.203 \\
ESS & 0.232 & $0.262^{*}$ & \\
\hline
\end{tabular}

TABLE 3: Correlation Between Demographic and Lifestyle Factors with Poor Sleep Quality in Groups 1, 2, and 3

* $=$ Significant $(p<0.05)$

BMI: body mass index; EM: electronic media, ESS: Epworth sleepiness score

\section{Discussion}

Our results showed that the majority of study subjects could be classified as intermediate type, followed by evening type and the least frequent one was the morning type. The results suggested that there was a substantial relationship between the chronotypes and the quality of sleep in the undergraduate students under study. The evening-type individuals showed worse quality of sleep when compared with the morning and intermediate types of individuals. Similar results were also found in other studies [20-21]. In this study, we found a high prevalence of poor quality of sleep among undergraduate students, corroborating previous researchers who also found the high prevalence of poor sleep quality among medical students. There are several pieces of evidence that medical students are more prone to sleep-wake disturbances [16]. This susceptibility to sleep disorders might be explained by the presence of a 
curricular load and school schedule disrespecting the morningness-eveningness balance among medical students [16]. Age and gender were significantly correlated with the poor sleep quality in the subjects of groups 1, 2, and 3. A study from Japan confirmed that girls had a longer sleep initiation time compared to boys [22]. Gender influences were present in sleep habits and sleep characteristics, but the results were inconsistent among studies [22-23]. Our results demonstrated that there are higher chances of smoking and alcohol use in evening type individual as compared to morning type individual. Similar findings were reported by previous studies [13, 15, 24]. An association between chronotypes and health-impairing lifestyles can be explained by different pathways. The first hypothetical pathway is innovation seeking [9]. Another pathway is through the association between chronotypes and markers of endogenous circadian rhythmicity [1, 14]. The misalignment of social and biological time might cause stress, which possibly leads to adaptive and maladaptive coping, such as tobacco smoking and alcohol drinking [13]. In the evening chronotype (electronic media use) and in the intermediate chronotype (duration of electronic media use) were significantly correlated with poor sleep quality. Use of social media sites was the most important factor that affected sleep quality. Studies have suggested that sleep duration and sleep quality were associated with social media and electronic media use [25-26]. It may be due to reduced total sleep time [27] or due to bright light emitted by the electronic media that may delay circadian rhythms [28]. In the present study, we also found that poor sleep quality was positively and significantly associated with the type of diet (vegetarian/non-vegetarian) in the evening and intermediate chronotypes. Reduced total sleep time and poor sleep quality were associated with a low intake of vegetables [29]. The present research highlighted that evening-type adolescents have a higher risk of healthimpairing lifestyles and also have an unhealthy eating pattern, characterized by a late night dinner. Replications of these findings are required in future research to explain the associations between circadian typology and health-impairing lifestyles. Future research should identify possible interventions according to the circadian typology to promote a healthy lifestyle and improve their quality of life. Our findings have public health importance for the large number of adolescents who have social jetlag and are sleep-deprived. We strongly advocate to promote the awareness in the medical field, as well as in the society, for the importance of circadian typology and the negative consequences of challenging our internal clock.

The present study was not without limitations. First, the present sample was limited to urban adolescents, and therefore, its generalization to a rural population is restricted. Second, this study was based only on self-report measures, which might be prone to memory and response biases.

\section{Conclusions}

The chronotypes demonstrated a significant association with the quality of sleep. Evening-type individuals showed a poor quality of sleep in comparison to the morning individuals, as well as the intermediate individuals. There was a high prevalence of poor quality of sleep and excessive diurnal sleepiness among the medical students. Furthermore, chronotypes also demonstrated the significant correlation of health-impairing lifestyles, such as tobacco smoking, alcohol drinking, and late night dinner, with sleep quality. From the observation of the impact of circadian biology among the medical students, there might be a better way to plan their daily activities, according to their chronotypes, benefiting not only their academic performance but also their quality of life.

\section{Additional Information}

\section{Disclosures}

Human subjects: Consent was obtained by all participants in this study. Ethical Committee of King George's Medical University (KGMU), Lucknow, India issued approval N/A. Animal subjects: All authors have confirmed that this study did not involve animal subjects or tissue. 
Conflicts of interest: In compliance with the ICMJE uniform disclosure form, all authors declare the following: Payment/services info: All authors have declared that no financial support was received from any organization for the submitted work. Financial relationships: All authors have declared that they have no financial relationships at present or within the previous three years with any organizations that might have an interest in the submitted work. Other relationships: All authors have declared that there are no other relationships or activities that could appear to have influenced the submitted work.

\section{Acknowledgements}

We are thankful to the Director of the Indian Council of Medical Research (ICMR), India. This study was not funded by any organisation. I also acknowledge all the authors for their contribution to the research work. I am especially indebted to the first-year medical undergraduates of King George's Medical University, India, who gave their valuable time for completing this research project.

\section{References}

1. Korczak AL, Martynhak BJ, Pedrazzoli M, et al.: Influence of chronotype and social zeitgebers on sleep/wake patterns. Braz J Med Biol Res. 2008, 41:914-19. 10.1590/S0100879X2008005000047

2. Susman EJ, Dockray S, Schiefelbein VL, et al.: Morningness/eveningness, morning-toafternoon cortisol ratio, and antisocial behavior problems during puberty. Dev Psychol. 2007, 43:811-22. 10.1037/0012-1649.43.4.811

3. Horne JA, Östberg O: A self-assessment questionnaire to determine morningness-eveningness in human circadian rhythms. Int J Chronobiol. 1976, 4:97-110.

4. Chelminski I, Petros TV, Plaud JJ, Ferraro FR: Psychometric properties of the reduced Horne and Östberg questionnaire. Pers Individ Dif. 2000, 29:469-78. 10.1016/S0191-8869(99)00208-1

5. Adan A, Almirall H: Horne and Östberg morningness-eveningness questionnaire: a reduced scale. Pers Individ Diff. 1991, 12:241-53. 10.1016/0191-8869(91)90110-W

6. Natale V, Cicogna P: Circadian regulation of subjective alertness in morning and evening ‘types'. Pers Individ Diff. 1996, 20:491-97. 10.1016/0191-8869(95)00213-8

7. Tankova I, Adan A, Buela-Casal G: Circadian typology and individual differences. A review . Pers Individ Diff. 1994, 16:671-84. 10.1016/0191-8869(94)90209-7

8. Paine SJ, Gander PH, Travier N: The epidemiology of morningness/eveningness: influence of age, gender, ethnicity, and socioeconomic factors in adults (30-49 years). J Biol Rhythms. 2006,21, 68-76. 10.1177/0748730405283154

9. Adan A, Natale V, Caci H, Prat G: Relationship between circadian typology and functional and dysfunctional impulsivity. Chronobiol Int. 2010, 27:606-19. 10.3109/07420521003663827

10. Fleig D, Randler C: Association between chronotype and diet in adolescents based on food logs. Eat Behav. 2009, 10:115-18. 10.1016/j.eatbeh.2009.03.002

11. Nakade M, Takeuchi H, Kurotani M, Harada T: Effects of meal habits and alcohol/cigarette consumption on morningness-eveningness preference and sleep habits by Japanese female students aged 18-29. J Physiol Anthropol. 2009, 28:83-90. 10.2114/jpa2.28.83

12. Schaal S, Peter M, Randler C: Morningness-eveningness and physical activity in adolescent . Int J Sport Exerc Psychol. 2010, 8:147-59. 10.1080/1612197X.2010.9671939

13. Wittmann M, Dinich J, Merrow M, Roenneberg T: Social jetlag: misalignment of biological and social time. Chronobiol Int. 2006, 23:497-509. 10.1080/07420520500545979

14. Giannotti F, Cortesi F, Sebastiani T, Ottaviano S: Circadian preference, sleep and daytime behaviour in adolescence. J Sleep Res. 2002, 11:191-99. 10.1046/j.1365-2869.2002.00302.x

15. Urbán R, Magyaródi T, Rigó A: Morningness-eveningness, chronotypes and health-impairing behaviors in adolescents. Chronobiol Int. 2011, 28:238-47. 10.3109/07420528.2010.549599

16. Rique GL, Fernandes Filho GM, Ferreira AD, de Sousa-Muñoz RL: Relationship between chronotype and quality of sleep in medical students at the Federal University of Paraiba, Brazil. Sleep Sci. 2014, 7:96-102. 10.1016/j.slsci.2014.09.004

17. Craig CL, Marshall AL, Sjöström M, et al.: International physical activity questionnaire: 12country reliability and validity. Med Sci Sports Exerc. 2003, 35:1381-95. 


\subsection{9/01.MSS.0000078924.61453.FB}

18. Buysse DJ, Reynolds CF 3rd, Monk TH, et al.: The Pittsburgh Sleep Quality Index: a new instrument for psychiatric practice and research. Psychiatry Research. 1988, 28:193-213. 10.1016/0165-1781(89)90047-4

19. Johns MW: A new method for measuring daytime sleepiness: the Epworth sleepiness scale . Sleep. 1991, 14:540-45. 10.1093/sleep/14.6.540

20. Selvi Y, Aydin A, Gulec M, et al.: Comparison of dream anxiety and subjective sleep quality between chronotypes. Sleep Biol Rhythms. 2012, 10:14-22. 10.1111/j.1479-8425.2011.00511.x

21. Barclay NL, Eley TC, Buysse DJ, et al.: Diurnal preference and sleep quality: same genes? A study of young adult twins. Chronobiol Int. 2010, 27:278-96. 10.3109/07420521003663801

22. Alexandru G, Michikazu S, Shimako H, et al.: Epidemiological aspects of self-reported sleep onset latency in Japanese junior high school children. J Sleep Res. 2006, 15:266-75. 10.1111/j.1365-2869.2006.00530.x

23. Natal CL, Lourenço TJ, Silva LA, et al.: Gender differences in the sleep habits of 11-13 year olds. Rev Bras Psiquiatr. 2009, 31:358-61. 10.1590/S1516-44462009000400013

24. Monk TH, Buysse DJ, Potts JM, et al.: Morningness-eveningness and lifestyle regularity. Chronobiol Int. 2004, 21:433-41. 10.1081/CBI-120038614

25. An J, Sun Y, Wan Y, et al.: Associations between problematic internet use and adolescents' physical and psychological symptoms: possible role of sleep quality. J Addict Med. 2014, 8:282-87. 10.1097/ADM.0000000000000026

26. Vernon L, Barber BL, Modecki KL: Adolescent problematic social networking and school experiences: The mediating effects of sleep disruptions and sleep quality. Cyberpsychol Behav Soc Netw. 2015, 18:386-92. 10.1089/cyber.2015.0107

27. Cain N, Gradisar M: Electronic media use and sleep in school-aged children and adolescents: a review. Sleep Med. 2010, 11:735-42. 10.1016/j.sleep.2010.02.006

28. Chang AM, Aeschbach D, Duffy JF, Czeisler CA: Evening use of light-emitting eReaders negatively affects sleep, circadian timing, and next-morning alertness. Proc Natl Acad Sci U S A. 2015, 112:1232-37. 10.1073/pnas.1418490112

29. Imaki M, Hatanaka Y, Ogawa Y, et al.: An epidemiological study on relationship between the hours of sleep and life style factors in Japanese factory workers. J Physiol Anthropol Appl Human Sci. 2002, 21:115-20. 10.2114/jpa.21.115 